\title{
Acculturation and Healthy Lifestyle Among Latinos With Diabetes
}

\author{
Arch G. Mainous III, $P b D^{1,2}$ \\ Vanessa A. Diaz, MD, MS ${ }^{1}$ \\ Mark E. Geesey, MS \\ 'Department of Family Medicine, Medical \\ University of South Carolina, Charleston \\ ${ }^{2}$ Department of Biostatistics, Bioinformatics \\ and Epidemiology, Medical University of \\ South Carolina, Charleston
}

\begin{abstract}
PURPOSE Latinos have a high prevalence of diabetes and disproportionately experience diabetic complications. We sought to examine the association of acculturation on healthy lifestyle habits among the Latino population in the United States with diabetes.

METHODS We studied Latino adults (aged $\geq 20$ years) with previously diagnosed diabetes in the 1999-2004 National Health and Nutrition Examination Survey (unweighted $N=467$; weighted $N=1,957,778$ ). Healthy lifestyle habits were those consistent with recommendations of the American Diabetes Association (ADA) regarding exercise, smoking, obesity, and diet. Acculturation was measured with the Short Acculturation Scale, a validated 5-item scale assessing use of the Spanish language. Logistic regression analyses of acculturation and healthy behaviors were computed controlling for access to care, demographics, and disease characteristics.

RESULTS In bivariate analyses, compared with their less-acculturated counterparts, individuals who were more-acculturated to any extent were less likely to have a higher fiber intake $(9.4 \%$ vs $35.4 \%, P=.001)$ and lower saturated fat intake $(17.2 \%$ vs $46.5 \%, P=.03)$. More-acculturated individuals were more likely to report leisure time exercise $(59.2 \%$ vs $19.3 \%, P<.001)$, whereas the proportion of individuals who smoked did not differ. In logistic regression analyses using the 1994 ADA dietary guidelines, more-acculturated individuals were less likely than their lessacculturated counterparts to meet dietary criteria for saturated fat consumption (odds ratio, 0.13; 95\% confidence interval [Cl], 0.04-0.41). When using the stricter 2006 ADA dietary guidelines, more-acculturated individuals were less likely to have recommended consumption of both saturated fat (odds ratio, $0.06 ; 95 \% \mathrm{Cl}, 0.02$ 0.20 ) and fiber (odds ratio, $0.19 ; 95 \% \mathrm{Cl}, 0.08-0.48$ ). Acculturation did not significantly influence exercise and smoking status in logistic regression analyses.
\end{abstract}

CONCLUSIONS These results suggest that acculturation among diabetic Latinos to the general us culture is associated with adoption of some less desirable dietary habits.

Ann Fam Med 2008;6:131-137. DOI: 10.1370/afm.814

\section{INTRODUCTION}

$\mathrm{D}$ iabetes has reached epidemic proportions in the United States. ${ }^{1}$

The deleterious outcomes of this disease can be decreased through appropriate ongoing management and treatment. ${ }^{2}$ Diabetes burden is not distributed equally throughout the population. In particular, Latinos have a higher prevalence of diabetes, more complications, and worse outcomes than non-Latino whites. ${ }^{3.7}$ Quality of care for diabetes among Latinos continues to lag behind that for non-Latino whites. ${ }^{8}$ Moreover, recent projections continue to predict an increasing prevalence of diabetes, particularly for Latinos. ${ }^{9}$

The US Census indicates that $14.5 \%$ of adult US residents are foreign-born. ${ }^{10}$ These immigrants, particularly minorities, may require interventions and modifications to the standard health care delivery system that differ from those of their ethnic counterparts who are born in the 
United States. Differences in diabetes prevalence and care among ethnic subgroups are associated with degree of acculturation. ${ }^{11,12}$ For example, Latinos who are less acculturated to the majority US culture tend to have a higher prevalence of diabetes and more complications. ${ }^{11,13}$

One aspect of successful diabetes management takes place outside of the physician's office and entails the patient adopting a healthy lifestyle. For patients with diabetes, a healthy lifestyle includes an appropriate diet and exercise. ${ }^{14,15}$ These self-management behaviors related to lifestyle may be particularly influenced by culturally specific attitudes and beliefs. ${ }^{16-18}$ In particular, some data suggest that acculturation for the general population of Latinos has been associated with suboptimal lifestyle choices, including a low intake of fruits and vegetables, a higher consumption of fats, and smoking. ${ }^{19}$ Acculturation among Latinos with diabetes to the mainstream US culture may thus influence the healthiness of their lifestyle, particularly depending on whether the mainstream society has a healthy lifestyle. The purpose of this study was to examine the association of acculturation with a healthy lifestyle among Latinos with diabetes in the United States.

\section{METHODS}

\section{Design}

We analyzed data from the 1999-2004 National Health and Nutrition Examination Survey (NHANES). Participants in this survey constitute a nationally representative sample of the noninstitutionalized US population. The NHANES design includes an oversampling of minorities and enables determination of population estimates. The NHANES program is relatively unusual compared with other nationally representative surveys in that it combines interviews with physical examinations and laboratory studies. More information on the methodology of the NHANES 1999-2004, including laboratory assessments, can be found at the National Center for Health Statistics Web site. ${ }^{20}$ NHANES data are not obtained using a simple random sample; rather, a complex, multistage, probability sampling design is used to select participants representative of the civilian, noninstitutionalized US population. Certain population subgroups are oversampled to increase the reliability and precision of estimates of health status indicators for these groups. The data are weighted to obtain population estimates of noninstitutionalized individuals in the United States.

\section{Sample}

The individuals analyzed in our study were Latino adults (aged $\geq 20$ years) with previously diagnosed diabetes. The NHANES contains a series of questions asked to ascertain an individual's race and ethnicity. Race and Hispanic ethnicity were addressed in separate questions. Latinos were the only group asked questions on language, allowing for assessments of acculturation. Interviews were conducted in both English and Spanish by trained bilingual interviewers. Respondents were free to switch between languages during the interview depending on which language they were most comfortable with in answering each question. Individuals were considered to have previously diagnosed diabetes if they answered affirmatively to a question of "Has a doctor ever told you that you have diabetes?" Analyses were based on 467 individuals, who represented a US population of $1,957,778$.

\section{Measures}

Acculturation Status

Acculturation is an indication of the cultural change of minority individuals to the majority culture. Acculturation measures vary widely depending on the construct used. We used 2 general constructs available in the NHANES 1999-2004 to assess acculturation: language and country of birth. Both of these have been used previously as proxies for acculturation. ${ }^{21-23}$

The degree of acculturation based on language was assessed using the Short Acculturation Scale (SAS), a 5 -item scale measuring use of the Spanish language that has good internal reliability (Cronbach $\alpha \geq 0.90$ ) and has been shown to be comparable to longer published acculturation scales. This scale has been validated in several Hispanic populations, with correlations seen between measured acculturation and number of generations living in the United States, length of time lived in the United States, age at the time of arrival in the United States, and self-reported acculturation. ${ }^{24}$ The scale consists of the following 5 questions: (1) In general, what language do you read and speak? (2) What was the language(s) you used as a child? (3) What language(s) do you usually speak at home? (4) In which language(s) do you usually think? and (5) What language(s) do you usually speak with your friends? The options for answering each question are "only Spanish," "more Spanish than English," "both equally," "more English than Spanish," and "only English." These responses were each scored on a scale from 0 to 4 , yielding a total score ranging from 0 to 20, with higher scores signifying greater acculturation. The acculturation score was used to define 5 categories representing a continuum of acculturation from Spanish language only (a score of 0), primarily Spanish (1-5), about equal use of Spanish and English (6-10), primarily English (11-15), and nearly all English (16-20). Because of the small size of the group with previously diagnosed diabetes, we classified their acculturation simply as any (1-20) vs none (0). 


\section{Healthy Lifestyle}

Measures of healthy lifestyle included smoking, obesity, exercise, and diet. Current smoking status was ascertained. Individuals with a body mass index (BMI) of $30 \mathrm{~kg} / \mathrm{m}^{2}$ or greater were considered obese, and those with a BMI from 25.0 to $29.9 \mathrm{~kg} / \mathrm{m}^{2}$ were considered overweight. Individuals were asked whether they participated in vigorous or moderate levels of leisure time physical activity; those who reported neither vigorous nor moderate physical activities were categorized as getting no exercise, whereas those who reported either or both were categorized as getting exercise. Respondents' diets were evaluated according to whether they met the 1994 ADA guidelines for individuals with diabetes in terms of daily fiber intake $(\geq 20 \mathrm{~g})$, cholesterol intake $(\leq 300 \mathrm{mg})$, and saturated fat intake ( $<10 \%$ of total calories). ${ }^{25}$ In addition, their diets were also assessed according to the 2006 ADA guidelines for fiber intake ( $\geq 14 \mathrm{~g}$ per 1,000 total calories), cholesterol intake $(<200 \mathrm{mg})$, and saturated fat intake ( $<7 \%$ of total calories) because these guidelines are more restrictive and are recommended based on current knowledge. ${ }^{26}$

\section{Demographic Characteristics and Access to Care}

We included the following demographic characteristics of respondents: sex, age (20-64 vs $\geq 65$ years), povertyincome ratio ( $<1.0 \mathrm{vs} \geq 1.0$ ), and education (high school graduate vs not). Access to care was assessed from whether the respondent had health insurance and a routine place for health care.

\section{Analysis}

Because the NHANES has a complex sampling design that makes the resulting sample representative of the noninstitutionalized US population, we were able to make nationally representative estimates. We used SUDAAN software (RTI International, Research Triangle Park, North Carolina) to account for the weighting and complex sampling design. We performed $\chi^{2}$ tests for bivariate relationships between acculturation, as measured both by the SAS score and by country of birth, and lifestyle measures.

We conducted multivariate analyses to investigate the relative odds that individuals with any level of acculturation (SAS score $>0$ ) practiced healthy habits, such as not smoking, getting exercise, and eating a healthy diet. Logistic regression analyses were computed and controlled for the potential confounding variables of age, sex, poverty, education, health insurance, having a routine place for care, number of years with diabetes, and having been told by a physician that one has retinopathy, hypertension, or high cholesterol. The ADA recommendations have recently changed regarding some dietary aspects of a healthy lifestyle, so we examined the relationship between acculturation and diet with both the recommendations in place at the time of data collection (1994 ADA guidelines) and the current recommendations (2006 ADA guidelines). Because socioeconomic status could potentially modify the relationship between acculturation and lifestyle, we investigated interaction effects between health insurance, poverty, and education and the SAS score in the models.

\section{RESULTS}

Depending on the measure used to assess acculturation, an estimated $36 \%$ to $69 \%$ of Latino individuals with diabetes nationally were more-acculturated (Table 1 ).

Latinos with diabetes who were less-acculturated according to SAS score were predominantly female, and they were significantly more likely that their more-acculturated counterparts to have annual incomes of less than $\$ 20,000$ and less likely to be high school graduates (Table 1). Less-acculturated individuals were also less likely to have private health insurance or a usual place for health care. There were no significant differences between the groups in terms of current smoking or BMI, but less-acculturated individuals exercised much less during leisure time. When using the 1994 ADA dietary guidelines, in bivariate analyses, dietary fiber and cholesterol intakes were not significantly different between the groups, but lessacculturated individuals were significantly more likely to get fewer than $10 \%$ of their daily calories from saturated fats. When using the 2006 ADA dietary guidelines, there still was no difference between the more-acculturated and less-acculturated groups with regard to dietary cholesterol intake; however, less-acculturated individuals were significantly more likely to have diets high in fiber and low in saturated fat. This effect was also apparent for acculturation as assessed by country of birth.

The relationships between the lifestyle characteristics and socioeconomic measures are shown in Table 2. The only significant differences were that compared with individuals who had graduated from high school, those who had not were less likely to exercise in their leisure time and more likely to have diets low in cholesterol and saturated fat.

The results of the logistic regression analyses indicated that when the SAS score was used as the indicator of acculturation, more-acculturated individuals were no more or less likely than less-acculturated individuals to be nonsmokers (odds ratio, $0.5 ; 95 \%$ confidence interval $[\mathrm{CI}], 0.17-1.72)$ or to engage in some leisure time exercise (odds ratio, 2.17; 95\% CI, 


\begin{tabular}{|c|c|c|c|c|c|c|c|}
\hline \multirow[b]{2}{*}{ Measure } & \multirow[b]{2}{*}{ Total } & \multicolumn{3}{|c|}{ SAS Score } & \multicolumn{3}{|c|}{ Country of Birth } \\
\hline & & 0 & $1-20$ & $P$ Value ${ }^{b}$ & Other & United States & $P$ Value $^{\mathrm{b}}$ \\
\hline $\begin{array}{l}\text { US population estimate, } \\
\text { No, } \%\end{array}$ & $1,957,778$ & 31.0 & 69.0 & - & 63.9 & 36.1 & - \\
\hline \multicolumn{8}{|l|}{ Demographics } \\
\hline Age $\geq 65$ years & 23.1 & 29.8 & 20.1 & .12 & 21.4 & 25.4 & .34 \\
\hline Male & 47.4 & 31.0 & 54.7 & .006 & 50.1 & 44.7 & .46 \\
\hline Annual family income $\geq \$ 20,000$ & 52.3 & 42.1 & 56.5 & .03 & 50.3 & 57.2 & .24 \\
\hline Poverty-income ratio $\geq 1.0$ & 72.5 & 68.5 & 74.1 & .42 & 74.0 & 70.5 & .53 \\
\hline High school graduate & 41.7 & 15.1 & 53.6 & $<.001$ & 31.6 & 61.9 & .01 \\
\hline \multicolumn{8}{|l|}{ Access to care } \\
\hline \multicolumn{8}{|l|}{ Health insurance } \\
\hline Public & 38.7 & 41.9 & 37.3 & .01 & 34.3 & 45.3 & .13 \\
\hline Private & 36.0 & 20.0 & 42.8 & & 34.0 & 41.8 & \\
\hline None & 25.3 & 38.1 & 19.9 & & 31.7 & 12.9 & \\
\hline Have a routine place for care & 94.9 & 89.2 & 97.4 & .01 & 93.7 & 97.3 & .17 \\
\hline \multicolumn{8}{|l|}{ Healthy lifestyle } \\
\hline Current smoker & 24.3 & 17.1 & 27.4 & .22 & 21.2 & 28.5 & .36 \\
\hline \multicolumn{8}{|l|}{ BMI } \\
\hline$<25 \mathrm{~kg} / \mathrm{m}^{2}$ & 19.0 & 16.5 & 20.1 & .88 & 17.5 & 20.8 & .93 \\
\hline $25-29.9 \mathrm{~kg} / \mathrm{m}^{2}$ & 36.8 & 37.4 & 36.6 & & 36.3 & 36.5 & \\
\hline$\geq 30 \mathrm{~kg} / \mathrm{m}^{2}$ & 44.2 & 46.1 & 43.3 & & 46.2 & 42.6 & \\
\hline \multicolumn{8}{|l|}{ Exercise } \\
\hline None & 53.2 & 80.7 & 40.8 & $<.001$ & 61.9 & 36.2 & .06 \\
\hline Moderate or vigorous & 46.8 & 19.3 & 59.2 & & 38.1 & 63.8 & \\
\hline \multicolumn{8}{|l|}{ Daily fiber intake } \\
\hline$\geq 20 \mathrm{~g}^{\mathrm{c}}$ & 31.2 & 33.2 & 30.3 & .69 & 32.1 & 32.3 & .98 \\
\hline$\geq 14 \mathrm{~g} / 1,000 \mathrm{kcal}^{\mathrm{d}}$ & 17.5 & 35.4 & 9.4 & .001 & 21.0 & 10.7 & .04 \\
\hline \multicolumn{8}{|l|}{ Daily cholesterol intake } \\
\hline$\leq 300 \mathrm{mgc}^{\mathrm{c}}$ & 59.8 & 65.0 & 57.5 & .28 & 66.9 & 48.5 & .03 \\
\hline$<200 \mathrm{mg}^{\mathrm{d}}$ & 43.5 & 50.4 & 40.3 & .23 & 47.8 & 38.0 & .18 \\
\hline \multicolumn{8}{|l|}{ Saturated fat intake } \\
\hline$<10 \%$ of total kcal & 53.0 & 76.3 & 42.4 & .001 & 62.2 & 34.0 & .001 \\
\hline$<7 \%$ of total $\mathrm{kcal}^{\mathrm{d}}$ & 26.4 & 46.5 & 17.2 & .03 & 34.1 & 11.2 & .003 \\
\hline \multicolumn{8}{|c|}{ SAS = Short Acculturation Scale; BMI = body mass index. } \\
\hline \multicolumn{8}{|c|}{ Note: Individuals were considered to be acculturated if they had an SAS score of 1 to 20 or if they were born in the United States. } \\
\hline $\begin{array}{l}\text { a Values are percentages of individuals } \\
\text { b Calculated by the } \chi^{2} \text { test. } \\
\text { ' The } 1994 \text { American Diabetes Associa } \\
\text { d The } 2006 \text { American Diabetes Associa }\end{array}$ & dietary recor & $\begin{array}{l}\text { dations fo } \\
\text { dations fo }\end{array}$ & tes. & & & & \\
\hline
\end{tabular}

0.85-5.53). When using the 1994 ADA dietary guidelines, more-acculturated individuals were significantly less likely to have diets low in saturated fats (Table 3). When using the 2006 ADA dietary guidelines, however, they were significantly less likely to have diets both high in fiber and low in saturated fat.

When country of birth was used as the indicator of acculturation, the associations between acculturation and healthy lifestyle were not as strong (Table 4). According to the 2006 criteria, acculturation was only associated with saturated fat intake, and that association indicated that individuals born outside the United States were more likely to have a diet low in saturated fat.
We found no evidence of an interaction between any of the socioeconomic indicators (health insurance, poverty, and education) and the SAS score in the models.

\section{DISCUSSION}

The results of this study suggest that although acculturation of Latinos to the mainstream US culture may be somewhat beneficial in terms of access to care and socioeconomic status, less-acculturated Latinos with diabetes tend to have healthier diets than their more-acculturated counterparts. Specifically, moreacculturated Latinos with diabetes were less likely to 


\begin{tabular}{|c|c|c|c|c|c|c|c|c|c|c|}
\hline \multirow{2}{*}{$\begin{array}{l}\text { Lifestyle } \\
\text { Indicator }\end{array}$} & \multicolumn{3}{|c|}{ Poverty-Income Ratio } & \multicolumn{3}{|c|}{ High School Graduate } & \multicolumn{4}{|c|}{ Health Insurance Status } \\
\hline & $<1.0$ & $\geq 1.0$ & $P$ Value ${ }^{b}$ & No & Yes & $P$ Value ${ }^{b}$ & Public & Private & None & $P$ Value $^{b}$ \\
\hline Smoker & 21.8 & 26.4 & .57 & 19.2 & 30.2 & .20 & 23.7 & 20.7 & 32.2 & .53 \\
\hline \multicolumn{11}{|l|}{ BMI } \\
\hline$<25 \mathrm{~kg} / \mathrm{m}^{2}$ & 30.2 & 14.5 & .29 & 18.1 & 19.4 & .98 & 21.4 & 15.3 & 18.5 & .10 \\
\hline $25-29.9 \mathrm{~kg} / \mathrm{m}^{2}$ & 35.6 & 38.7 & & 36.7 & 35.9 & & 42.8 & 42.4 & 25.0 & \\
\hline$\geq 30 \mathrm{~kg} / \mathrm{m}^{2}$ & 34.2 & 46.8 & & 45.2 & 44.7 & & 35.8 & 42.3 & 56.5 & \\
\hline \multicolumn{11}{|l|}{ Exercise } \\
\hline None & 56.5 & 50.2 & .41 & 66.3 & 33.8 & .009 & 60.4 & 37.5 & 64.5 & .06 \\
\hline Any & 43.5 & 49.8 & & 33.7 & 66.2 & & 39.6 & 62.5 & 35.5 & \\
\hline \multicolumn{11}{|l|}{ Fiber intake } \\
\hline$\geq 20 \mathrm{~g}^{c}$ & 29.2 & 35.3 & .36 & 26.7 & 39.7 & .19 & 30.1 & 30.8 & 42.1 & .35 \\
\hline$\geq 14 \mathrm{~g} / 1,000 \mathrm{kcal}^{\mathrm{d}}$ & 21.2 & 16.2 & .30 & 22.0 & 11.1 & .06 & 17.0 & 12.6 & 25.1 & .29 \\
\hline \multicolumn{11}{|c|}{ Daily cholesterol intake } \\
\hline$\leq 300 \mathrm{mg}^{\mathrm{c}}$ & 63.1 & 59.1 & .63 & 70.2 & 46.7 & .02 & 69.7 & 30.9 & 48.0 & .09 \\
\hline$<200 \mathrm{mg}^{\mathrm{d}}$ & 46.8 & 43.8 & .69 & 50.5 & 35.7 & .06 & 52.2 & 45.6 & 30.4 & .13 \\
\hline \multicolumn{11}{|l|}{ Saturated fat intake } \\
\hline$<10 \%$ total kcal & 49.7 & 51.5 & .88 & 61.5 & 39.3 & .02 & 52.1 & 52.8 & 46.9 & .91 \\
\hline$<7 \%$ total kcald & 24.1 & 26.1 & .81 & 28.4 & 22.7 & .36 & 22.3 & 31.3 & 20.8 & .48 \\
\hline \multicolumn{11}{|l|}{$\mathrm{BMI}=$ body mass index. } \\
\hline $\begin{array}{l}\text { a Values are percentages } \\
\text { b Calculated with the } \chi^{2} \\
\text { 'The } 1994 \text { American Di } \\
\text { d The } 2006 \text { American Di }\end{array}$ & dividuals & tary & ndat & ett & & & & & & \\
\hline
\end{tabular}

Table 3. Relative Odds of Healthy Dietary Indicators for Latino Individuals With Diabetes Having an SAS Score of 1 to 20 vs an SAS Score of 0

\begin{tabular}{lc}
\hline Dietary Indicator & $\begin{array}{c}\text { Odds Ratio } \\
\text { (95\% Confidence Interval) }{ }^{\mathbf{a}}\end{array}$ \\
\hline 1994 ADA dietary recommendations & $0.58(0.26-1.30)$ \\
Daily fiber intake $\geq 20 \mathrm{~g}$ & $1.30(0.65-2.56)$ \\
Daily cholesterol intake $\leq 300 \mathrm{mg}$ & $0.13(0.04-0.41)$ \\
Saturated fat intake $<10 \%$ of total kcal & \\
2006 ADA dietary recommendations & $0.19(0.08-0.48)$ \\
Daily fiber intake $\geq 14 \mathrm{~g} / 1,000 \mathrm{kcal}$ & $0.70(0.38-1.30)$ \\
Daily cholesterol intake $<200 \mathrm{mg}$ & $0.06(0.02-0.20)$ \\
Saturated fat intake $<7 \%$ of total kcal & \\
\hline SAS = Short Acculturation Scale; ADA = American Diabetes Association. \\
all models are adjusted for age-group, sex, family income, education level, health insurance, \\
years with diabetes, whether the individual had a routine place for health care, and whether the \\
individual had retinopathy, hypertension, and high cholesterol. \\
\hline
\end{tabular}

of perceived behavioral control, as they are unable to develop strategies to protect these beneficial traditional habits. ${ }^{27-29}$

Acculturation has been linked to the likelihood of developing diabetes, as well as to quality of care, with more-acculturated individuals receiving better care. ${ }^{8,13}$ Little research has focused on the impact of acculturation on diet among Latinos with diabetes, however. Delivery of culturally competent health care by professionals has been discussed at length, but evidence suggests that self-management of diabetes in modern US society may be difficult for Latino patients as they struggle to decide which traditional behaviors to retain and which new ones to adopt. ${ }^{28}$

Our findings suggest that an under-

have diets higher in fiber and lower in saturated fat; moreover, this effect was more apparent when using the stricter ADA dietary criteria put forth in 2006. These differences suggest that adopting an "American" diet may not be a desirable change in behavior. This finding is especially pertinent in light of previous studies demonstrating that Latinos have difficulty in maintaining healthy traditional habits while adapting to the mainstream culture, which may be due to a lack standing of the complexities in providing culturally competent care must include an understanding not just of where the patient is coming from culturally, but where they are now. Having a medical home with continuity of care may help to ensure that the patient-physician relationship is intact and that the physician can understand factors that may be changing in the patient's ability to adhere to treatment or lifestyle recommendations. Consequently, 


\begin{tabular}{|c|c|}
\hline Dietary Indicator & $\begin{array}{l}\text { Odds Ratio } \\
\text { (95\% Confidence Interval) }^{\text {a }}\end{array}$ \\
\hline \multicolumn{2}{|l|}{1994 ADA dietary recommendations } \\
\hline Daily fiber intake $\geq 20 \mathrm{~g}$ & $1.10(0.49-2.48)$ \\
\hline Daily cholesterol intake $\leq 300 \mathrm{mg}$ & $0.48(0.22-1.02)$ \\
\hline Saturated fat intake $<10 \%$ of total kcal & $0.38(0.13-1.08)$ \\
\hline \multicolumn{2}{|l|}{2006 ADA dietary recommendations } \\
\hline Daily fiber intake $\geq 14 \mathrm{~g} / 1,000 \mathrm{kcal}$ & $0.63(0.29-1.40)$ \\
\hline Daily cholesterol intake $<200 \mathrm{mg}$ & $0.58(0.28-1.23)$ \\
\hline Saturated fat intake $<7 \%$ of total kcal & $0.15(0.04-0.54)$ \\
\hline
\end{tabular}

an initial lack of health insurance or disruption of health insurance coverage is a big problem in socially disadvantaged populations because of its impact on this continuity and having a medical home, and corresponding delivery of quality care. ${ }^{30}$

There are several limitations to this study. First, the data set is cross-sectional, which limits the ability to make inferences about causality. The NHANES data do, however, allow us to generate population estimates for Latinos with diabetes in the US population. Second, we used both a general measure of acculturation (being born in the United States) and a standard scale that represents acculturation based on language (SAS score), and the latter was significantly associated with several indicators of healthy lifestyle. It is possible, however, that because acculturation includes multiple factors regarding behaviors and beliefs, our measure was too crude and simplistic to appropriately capture nuances of culture. Third, the question regarding physiciandiagnosed diabetes, which was the basis for including individuals in the study, does not discriminate between individuals told that they had type 1 diabetes and individuals told that they had type 2 diabetes; ${ }_{i}$ however, the lifestyle questions we used are applicable to individuals with either type of diabetes. Fourth, although the NHANES allowed us to look at the current behaviors and acculturation of Latinos generally, the survey questions were limited regarding background characteristics of the subset of Latino immigrants. Consequently, we were unable to investigate questions such as whether immigrants from rural areas of Mexico differ in their behaviors from those hailing from urban areas. Fifth, in the bivariate analyses, more-acculturated individuals were more likely to engage in leisure time exercise than their less-acculturated counterparts. This finding does not necessarily mean that less-acculturated individuals do not engage in exercise, but rather that they may not use their leisure time for exercise. Individuals working in more physical and manual labor occupations may seek out less vigorous leisure time pursuits. For many, the importance of exercising after work may take a lower priority than resting from a workday considerably longer than 8 hours that involves physical activity. Even though we could not measure exercise obtained during leisure time vs on the job, once we controlled for demographic characteristics, the relationship between acculturation and leisure time exercise was no longer significant.

In conclusion, the common assumption that ethnic minority populations will always benefit from acculturation to mainstream US society does not appear to hold true in the case of healthy lifestyle behaviors among Latinos with diabetes. This study adds to other evidence that aspects of acculturation may be maladaptive. ${ }^{19}$ Dietary habits of Latinos with diabetes who are lessacculturated to US society are healthier than those of Latinos who are more-acculturated. It is important to understand the complex process of acculturation as we focus on developing culturally sensitive health care programs, in order to encourage patients to maintain healthy traditional behaviors while simultaneously adopting beneficial aspects of the mainstream culture.

To read or post commentaries in response to this article, see it online at http://www.annfammed.org/cgi/content/full/6/2/131.

Key words: Acculturation; culture; Latinos; immigrants; diabetes mellitus; lifestyle; health behavior; diet; exercise

Submitted July 5, 2007; submitted, revised, September 21, 2007; accepted October 8, 2007.

Funding support: This project was supported in part by grant 1 P3OAG021677 from the National Institute on Aging and by grants from the Robert Wood Johnson Foundation.

\section{References}

1. Cowie CC, Rust KF, Byrd-Holt DD, et al. Prevalence of diabetes and impaired fasting glucose in adults in the U.S. population: National Health and Nutrition Examination Survey 1999-2002. Diabetes Care. 2006;29(6):1263-1268.

2. Vijan S, Stevens DL, Herman WH, Funnell MN, Staniford CJ. Screening, prevention, counseling, and treatment for the complications of type II diabetes mellitus. Putting evidence into practice. J Gen Intern Med. 1997;12(9):567-580.

3. Cowie CC, Rust KF, Byrd-Holt D, et al. Prevalence of diabetes and impaired fasting glucose in adults-United States, 1999-2000. MMWR Morb Mortal W/kly Rep. 2003;52(35):833-837. 
4. Pugh JA, Stern MP, Haffner SM, et al. Excess incidence of treatment of end-stage renal disease in Mexican Americans. Am J Epidemiol. 1988;127(1):135-144

5. Harris MI, Eastman RC, Cowie CC, et al. Racial and ethnic differences in glycemic control of adults with type 2 diabetes. Diabetes Care. 1999;22(3):403-408.

6. Karter AJ, Ferrara A, Liu JY, et al. Ethnic disparities in diabetic complications in an insured population. JAMA. 2002;287(19):2519-2527.

7. Resnick HE, Foster GL, Bardsley J, Ratner RE. Achievement of American Diabetes Association Clinical Practice Recommendations among U.S. adults with diabetes, 1999-2002: the National Health and Nutrition Examination Survey. Diabetes Care. 2006;29(3):531-537.

8. Mainous AG III, Diaz VA, Koopman RJ, Everett CJ. Quality of care for Hispanic adults with diabetes. Fam Med. 2007;39(5):351-356.

9. Mainous AG III, Baker R, Koopman RJ, et al. Impact of the population at risk of diabetes on projections of diabetes burden in the United States: an epidemic on the way. Diabetologia. 2007;50(5):934-940.

10. Larsen LJ. The Foreign-Born Population in the United States: 2003. Current Population Reports, P20-551. Washington, DC: US Census Bureau; 2004.

11. Mainous AG III, Majeed A, Koopman RJ, et al. Acculturation and diabetes among Hispanics: evidence from the 1999-2002 National Health and Nutrition Examination Survey. Public Health Rep. 2006;121(1):60-66

12. Mainous AG III, Baker R, Majeed A, et al. English language skills and diabetes and hypertension among foreign-born South Asian adults in England. Public Health Rep. 2006;121(3):331-336.

13. Hazuda HP, Haffner SM, Stern MP, Eifler CW. Effects of acculturation and socioeconomic status on obesity and diabetes in Mexican Americans: the San Antonio Heart Study. Am J Epidemiol. 1988;128(6):1289-1301

14. Bantle JP, Wylie-Rosett J, Albright AL, et al. Nutrition recommendations and interventions for diabetes-2006: a position statement of the American Diabetes Association. Diabetes Care. 2006;29(9): 2140-2157.

15. Roberts CK, Won D, Pruthi S, Lin SS, Barnard RJ. Effect of a diet and exercise intervention on oxidative stress, inflammation and monocyte adhesion in diabetic men. Diabetes Res Clin Pract. 2006;73(3):249-259

16. Lai WA, Chie W-C, Lew-Ting C-Y. How diabetic patients' ideas of illness course affect non-adherent behaviour: a qualitative study. $\mathrm{Br}$ J Gen Pract. 2007;57(537):296-302
17. Serour M, Alqhenaei H, Al-Saqabi S, Mustafa A-R, Ben-Nakhl A Cultural factors and patients' adherence to lifestyle measures. $\mathrm{Br}$ J Gen Pract. 2007;57(537):291-295.

18. Hjelm K, Nyberg P, Isacsson A, Apelqvist J. Beliefs about health and illness essential for self-care practice: a comparison of migrant Yugoslavian and Swedish diabetic females. J Adv Nurs. 1999;30(5):1147-1159.

19. Perez-Escamilla $R$, Putnik P. The role of acculturation in nutrition, lifestyle, and incidence of type 2 diabetes among Latinos. J Nutr. 2007;137(4):860-867.

20. Centers for Disease Control and Prevention (US), National Center for Health Statistics. National Health and Nutrition Examination Survey. NHANES 1999-2004. http://www.cdc.gov/nchs/about/ major/nhanes/nhanes99-02.htm. Accessed Apr 24, 2007.

21. Mainous AG III. Self-concept as an indicator of acculturation in Mexican Americans. Hisp J Behav Sci. 1989;11(2):178-189.

22. Griffith J. Relationship between acculturation and psychological impairment in adult Mexican Americans. Hisp J Behav Sci. 1983; 5(3):431-459.

23. Nesdale D. Acculturation attitudes and the ethnic and host-country identification of immigrants. J Appl Soc Psychol. 2002;32(7):14881507.

24. Marin G, Sabogal F, Van Oss Marin B, Otero-Sabogal R, PerezStable EJ. Development of a short acculturation scale for Hispanics. Hisp J Behav Sci. 1987;9(2):183-205.

25. American Diabetes Association. Nutrition recommendations and principles for people with diabetes mellitus. Diabetes Care. 1994;17(9):519-522.

26. American Diabetes Association. Nutrition recommendations and interventions for diabetes-2006: a position statement of the American Diabetes Association. Diabetes Care. 2006;29(9):2140-2157.

27. Ajzen I, Fishbein M. Understanding Attitudes and Predicting Social Behavior. Englewood Cliffs, NJ: Prentice Hall; 1980.

28. Diaz VA, Mainous AG III, Pope C. Cultural conflicts in the weight loss experience of overweight Latinos. Int J Obes. 2007;31(2):328-333.

29. Devlin H, Roberts M, Okaya A, Xiong YM. Our lives were healthier before: focus groups with African American, American Indian, Hispanic/Latino, and Hmong people with diabetes. Health Promot Pract. 2006;7(1):47-55.

30. Starfield B, Shi L. The medical home, access to care, and insurance: a review of evidence. Pediatrics. 2004;113(5 Suppl):1493-1498. 